\title{
REVIEW
}

\section{Gene Therapy Progress and Prospects: Cystic fibrosis}

\author{
U Griesenbach, S Ferrari, DM Geddes and EWFW Alton \\ Department of Gene Therapy, National Heart and Lung Institute, Imperial College, Faculty of Medicine, London, UK
}

\begin{abstract}
Since the cloning of the cystic fibrosis gene (CFTR) in 1989, 18 clinical trials have been carried out, including five in the 2 years reviewed here. Most trials demonstrated proof-ofprinciple for gene transfer to the airway. However, gene transfer efficiency with each of the three gene transfer agents (adenovirus $(A d)$, adeno-associated virus 2 (AAV2)
\end{abstract}

and cationic liposomes) was low, and most likely insufficient to achieve clinical benefit. Here, we will review the clinical and pre-clinical progress for the last 2 years (2000-2001) and briefly speculate on future prospects for the next 2 in CF gene therapy.

Gene Therapy (2002) 9, 1344-1350. doi:10.1038/sj.gt.3301791

Keywords: cystic fibrosis; gene therapy; airway gene transfer

\section{In brief}

\section{Progress}

- Five clinical trials for CF have been carried out between 2000 and 2001

- Encouraging progress has been made to overcome some of the extracellular barriers to airway gene transfer, such as mucus and the glycocalyx

- Important intracellular barriers, such as cytoplasmic nucleases and the nuclear membrane have been identified and first attempts been made to overcome these barriers

- Viruses that recognise receptors on the apical surface of airway epithelial cells have been identified

- Targeted receptor-mediated endocytosis of synthetic vectors has increased transfection efficiency of airway epithelial cells

- Repeated administration of viral vectors, but not non-viral vectors, remains a significant problem

- Expression cassettes have been improved to enable prolonged transgene expression
- Intravenous and in utero gene delivery have been evaluated

- Genomic gene repair, mRNA trans-splicing and antisense approaches have been introduced for $\mathrm{CF}$

\section{Prospects}

- Integrating viral and non-viral GTAs will prolong gene expression in the airways and expression will be regulated through on/off cassettes

- Repeated administration of viral vectors will be improved

- Physical methods of gene delivery will be developed and will overcome some of the barriers to topical gene transfer

- Better regulatory elements will be developed

- Stem cell gene therapy will advance

- Better animal models will begin to be developed

- New imaging techniques to monitor the success and effect of gene transfer and more clinically relevant endpoint assay will be developed

\section{Five clinical trials for CF have been carried out between 2000 and 2002}

Three out of the five trials used liposome-mediated gene transfer strategies, one trial used Ad2 and one AAV2. Noone et al transfected the nasal epithelium of CF patients and was able to detect vector-specific DNA up to 10 days after gene transfer, but not mRNA or functional correction of the CFTR defect. ${ }^{1}$ The discrepancy between this trial and previously published nose trials that were able to detect CFTR mRNA and partial correction of the CFTR defect, is likely due to the lower transfection efficiency of the cationic lipid used. In a dose-escalating safety trial, liposome DNA complexes were aerosolised into the lungs of CF subjects, half of which, had a transient fever, muscle and joint pain shortly after

Correspondence: U Griesenbach, Department of Gene Therapy, Faculty of Medicine, National Heart and Lung Institute, Imperial College, London, SW3 6LR, UK
liposome/DNA administration. ${ }^{2}$ This was attributed to an immunological response against the liposome/DNA complexes; similar results were previously reported in a lung trial, using the same liposome/DNA formulation. Interestingly, Hyde et al demonstrated for the first time, that liposome/DNA complexes could be successfully readministered to the nose of CF patients. Each subject received three doses, administered 4 weeks apart and samples were analysed 4 days after each treatment. Six out of 10 treated subjects were positive for CFTR gene transfer after each dose. ${ }^{3}$

Perricone et al reported the results of a phase I clinical trial, in which recombinant adenovirus (Ad2/CFTR) was administered through bronchoscopic instillation or aerosolisation to the lungs of CF patients. In contrast to previous studies, the authors carefully determined that inefficient gene expression was due to the very low transduction efficiency of Ad in human airways. In addition, the vast majority of transfected cells (97\%), did not appear to be epithelial cells. ${ }^{4}$ The authors therefore 
concluded that further improvements in Ad vector design are urgently required. An AAV2 vector was nebulised into the lung of CF subjects to assess delivery and safety. AAV2 administration to the lung appeared to be safe and vector genome was detected, at the highest dose, up to 30 days after administration. However, vector-specific mRNA could not be detected and therefore evidence for gene transfer was not provided. ${ }^{5}$

In summary, these new studies consolidate the view that proof-of-principle of gene transfer can be demonstrated in some, but not all studies, but that gene transfer efficacy is currently insufficient to warrant phase II/III trials. Thus, significant improvements in all aspects of gene transfer need to be made.

\section{Encouraging progress has been made to overcome some of the extracellular barriers to airway gene transfer, such as mucus and the glycocalyx}

A major function of the airway epithelium is to prevent uptake of foreign materials, including gene transfer agents (GTAs). For this purpose several very effective extracellular barriers, such as mucus, the gycocalyx, tight junctions and mucociliary clearance have evolved (Figure 1). Mucus, for example, reduces the transfection efficiency of most viral and non-viral gene transfer agents. However, transfection efficiency could be increased through pre-treatment with mucolytics or the antichiolinergic drug glycopyrolate in vitro and in vivo. ${ }^{6}$ In addition to mucus, sputum and bronchoalveolar lavage fluid recovered from CF patients have been shown to inhibit liposome, ${ }^{7}$ adenovirus ${ }^{8}$ and AAV-mediated gene transfer efficiency. ${ }^{9}$ Although recombinant DNase reduced sputum viscoelasticity and improved nanosphere migration in vitro, ${ }^{10}$ the effect of DNase on gene transfer in vivo is unclear. To avoid the confounding effect of sputum in vivo, gene transfer should ideally be studied in CF children, before their lungs become filled with secretions. Indirect evidence has indicated, that the glycocalyx is also a barrier to gene transfer and that neu-

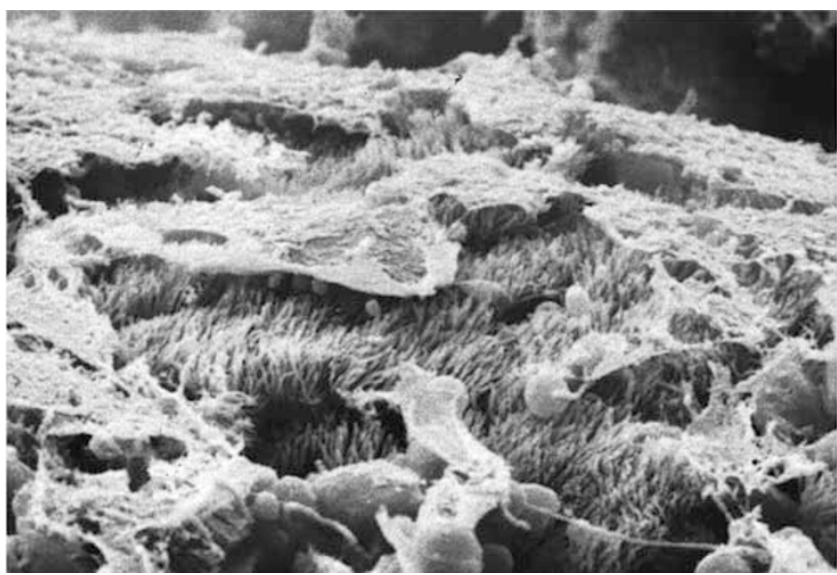

Figure 1 Scanning electron micrograph of human airway epithelium showing fields of cilia covered at their tips by flakes of mucus. In hypersecretory disease the mucus usually forms a continuous sheet or 'blanket' overlying the cilia (supplied by courtesy of Professor Peter Jeffery, Imperial College, London, UK). roaminidase enhances Ad transfection of polarised cells in vitro. ${ }^{11}$

Receptors for Ad and AAV2 are located on the basolateral membrane of human airway epithelial cells and uptake of non-viral gene transfer agents also appears to be enhanced on the basolateral site, due to the presence of heparan receptors and a higher endocytosis rate. The effect of several tight junction 'openers', including EGTA ${ }^{12,13}$ anti-E-cadherin antibody, ${ }^{14}$ sodium caprate, ${ }^{15}$ a blend of sucrose, mannitol and Pluronic F68 ${ }^{16}$ or perfluorochemical ${ }^{17,18}$ on gene transfer has been studied in vitro and in vivo. Although a 10 - to 50 -fold increase in virus- and non-virus-mediated gene transfer was generally seen in vitro, it is unlikely that the opening of tight junctions, even if transient, will be clinically applicable, given that the airways of most CF patients are heavily colonised with bacteria.

In summary, although encouraging progress has been made partially to overcome some extracellular barriers, it is unlikely that these strategies on their own will significantly improve gene transfer in the human airways. However, they may prove beneficial in combination with other strategies (see below).

\section{Targeted receptor-mediated endocytosis of synthetic vectors has increased the transfection efficiency of airway epithelial cells}

Liposomes and other synthetic vectors are also unable to transfect airway epithelial cells efficiently via the apical membrane. Targeted receptor-mediated endocytosis may increase gene transfer and proof-of-principle for this approach has recently been provided by Ziady et al, who have shown that targeting of the serpin enzyme complex receptor (sec-R) increases gene transfer to the nasal epithelium of mice. ${ }^{19}$ The sec-R ligand was linked to the CFTR plasmid via a poly-L-lysine bridge, and this formulation partially corrected the CFTR defect (chloride efflux) in the nose of CFTR knockout mice. Most remarkably, it was demonstrated for the first time in vivo that CF-related secondary defects (nitric oxide synthase-2 and sodium hyper-absorption) were partially correctable through gene therapy as well. ${ }^{19}$ Targeting of sec- $\mathrm{R}$ has been successful in increasing airway gene transfer and several groups are currently trying to identify new peptides that bind to airway epithelial cells and promote targeted receptor-mediated endocytosis, using phage display libraries. ${ }^{20}$

\section{Viruses that recognise receptors on the apical surface of airway epithelial cells have been identified}

As mentioned above, the density of receptors for Ad and AAV2 on the apical membrane of human airway epithelial cells is low, which explains in part the inefficient transfection efficiency. Multiple approaches have been studied to overcome this problem. Firstly, several new recombinant viruses have been identified, that appear to be able to enter epithelial cells via the apical membrane efficiently. In addition to respiratory syncytial virus (RSV), ${ }^{21}$ Sendai virus $(\mathrm{SeV})^{22}$ has recently been shown to transfect bronchial epithelial cells efficiently in vivo. Two 
days after infection with $\mathrm{SeV} 80 \%$ of airway epithelial cells expressed the $\beta$-galactosidase transgene (Figure 2a). $\mathrm{SeV}$ uses binding to cholesterol and sialic acid as receptors, which are both present at the apical membrane of airway epithelial cells. In addition, $\mathrm{SeV}$ is not greatly inhibited by mucus. AAV entry into airway epithelial cells appears to be serotype-specific and AAV5 has been shown to transfect airway epithelial cells five times more efficiently than AAV2. ${ }^{23,24} \mathrm{AAV}$ in general is a very promising gene transfer agent. Not only does the virus maintain prolonged transgene expression due to integration or concatemerisation of the vector genome, but it is also thought that the virus might be less immunogenic, due to the fact that it does not appear to transduce antigenpresenting dendritic cells. Some reports have suggested that AAV could be repeatedly administered to the lung. However, this possibility has recently been ruled out unless different serotypes or some form of immunosuppression are used. ${ }^{25}$ An important limitation of AAV as gene transfer vector for CF has been its limited packaging capacity. However, it has recently been demonstrated that this problem can be overcome by using trans-splicing or overlapping vectors ${ }^{26}$ or by shortening the CFTR cDNA. ${ }^{27}$

Secondly, proof-of-principle has been established that adeno and AAV vectors can be retargeted to apical surface receptors, such as the bradykinin, ${ }^{28}$ the urokinase plasminigen $^{29}$ and the $\mathrm{P}_{2} \mathrm{Y}_{2}$-purinoreceptor ${ }^{30}$ in vitro. However, it remains to be established if these approaches will increase transfection efficiency in vivo.

A third strategy involves systematic pseudotyping of enveloped virus with different envelope glycoproteins. Glycoproteins from viruses that naturally infect the airway epithelial cells, such as human coronavirus $229 \mathrm{E},{ }^{31}$ influenza A strains ${ }^{32}$ and members of the Filovirus family, such as Marburg or Ebola virus, are good candidates. Pseudotyping of lentiviral vectors such as the human, feline or equine immunodeficiency virus is a particularly attractive approach. Lentiviral vectors have the ability to integrate into the genome of dividing and non-dividing, differentiated cells. Expression is therefore prolonged when compared with episomally maintained vectors and may last forever, if integration into a stem cell occurred. Kobinger et al have recently demonstrated that an Ebola- pseudotyped HIV vector efficiently transfects airway epithelial cells in vivo. ${ }^{33}$ Most interestingly, expression was low 7 days after administration, but strong expression was visible in airway epithelium and submucosal gland cells 28 days after transfection (30\% tracheal epithelium expressed $\beta$-galactosidase). This persisted at least until day 63 (24\% of the tracheal epithelium expressed a $\beta$ galactosidase reporter gene) (Figure 2b). Currently largescale, high titre production of pseudotyped lentiviral vectors is technically difficult and a critical limiting factor.

The identification of viruses that are capable of efficiently transfecting the airway epithelium via the apical membrane has been one of the most exciting findings in the last 2 years. Although big problems such as repeated administration still exist, proof-of-principle studies, demonstrating that the primary CFTR defect can be consistently corrected in humans, should now become possible.

\section{Repeated administration of viral vectors, but not non-viral vectors, remains a significant problem}

Transgene expression will remain transient, unless lung repopulating stem cells can be targeted with a stably maintained vector. The treatment of CF with gene therapy will therefore require repeated administrations of gene transfer agents, which is a particular problem for viruses. Strategies such as administration of immunosuppressants and corticosteroids, and treatments aimed at transiently blocking $\mathrm{CD}^{+} \mathrm{T}$ cells ${ }^{34}$ have been evaluated for repeated administration of adenovirus. However, the success of these strategies has been limited. In general, repeated administration was possible a few times, but ultimately lead to reduced and finally absent transgene expression. The only exception so far is a report by Kolb et al, who demonstrated that administration of the steroid budesonide enabled adenovirus to be re-administered at least five times without loss of transfection efficiency. ${ }^{35}$ Importantly, treatment with an anti-CD40 ligand monoclonal antibody did not prevent a virus specific antibody response in non-human primates. ${ }^{34}$

Another and perhaps more promising approach is
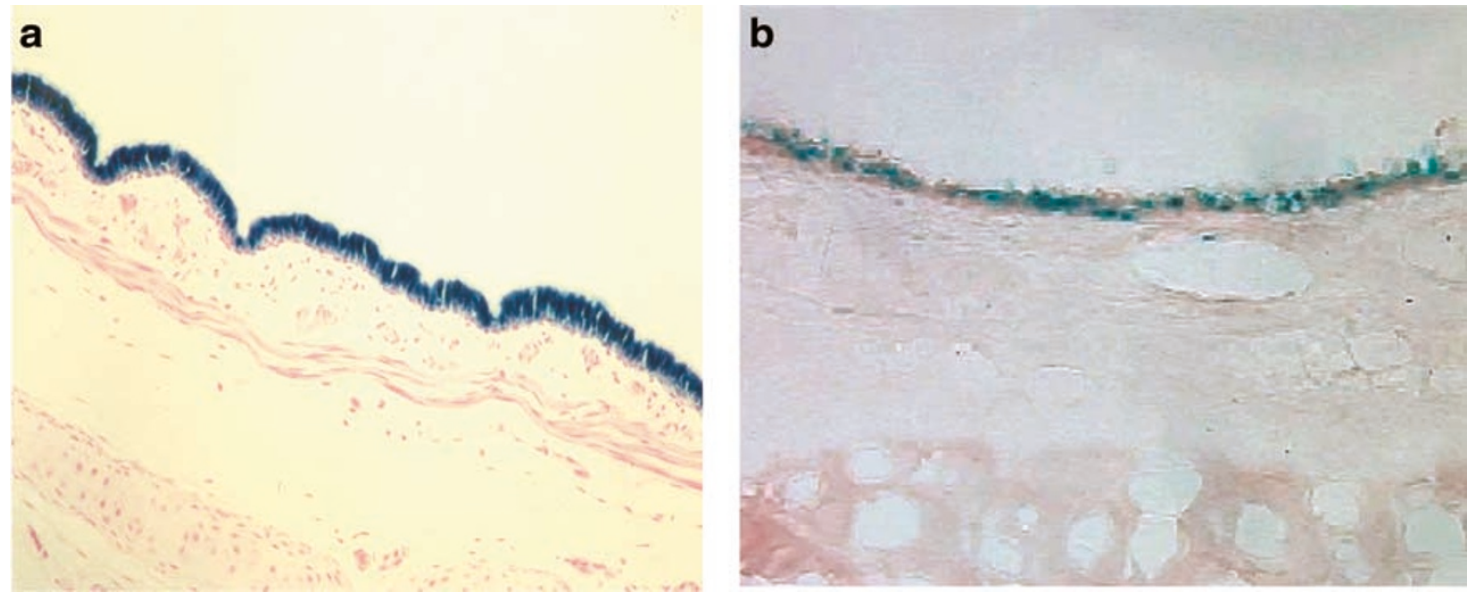

Figure 2 Viral gene transfer to the lung. (a) Recombinant Sendai virus (SeV) mediated $\beta$-galactosidase expression 48 h after gene transfer. ${ }^{48}$ (b) Filovirus pseudotyped lentivirus-mediated $\beta$-galactosidase expression 63 days after gene transfer (with permission from Ref. 33). 
based on generating a 'stealth virus', which is invisible to the immune system, by coating the virus capsid with polyethylene glycol (PEG). PEGylation of the virus capsid reduced cytotoxic $\mathrm{T}$ cells and antibody production and significantly prolonged transgene expression from 4 to 42 days. Repeated administration of the adenovirus modified with the same PEG was not successful, however, when different PEG formulations were used, significant transgene expression was detected after repeat administration. ${ }^{36}$

Although, it has been demonstrated that repeated administration of liposome/DNA complexes to the nose of CF patients is possible, ${ }^{3}$ there have been concerns raised regarding the inflammatory components of bacterial DNA. ${ }^{2}$ The abundance of unmethylated CpG motifs in the bacterial plasmid DNA may at least in part be responsible for the inflammatory response. Several strategies are currently being explored to decrease these unwanted properties, such as (1) methylation of $\mathrm{CpG}$ sequences; (2) reduction of the $\mathrm{CpG}$ frequency by eliminating non-essential regions or by site-directed mutagenesis; and (3) the use of specific inhibitors of the CpG signalling pathway, such as chloroquine or quinacrine. ${ }^{37}$

In summary, progress in enabling repeated administration of viruses has been rather slow and concerns regarding viral and non-viral GTA-induced inflammation have been raised. A more detailed understanding of the immune responses against viral and non-viral GTAs is therefore crucial for further improvements in gene transfer.

\section{Important intracellular barriers including cytoplasmic nucleases and the nuclear membrane have been identified and first attempts been made to overcome these barriers}

Intracellular barriers limiting transgene expression have also been identified. Endosomal and cytoplasmic degradation are a particular problem for most non-viral gene transfer agents. In the cytoplasm, $\mathrm{Ca}^{2+}$-sensitive cytosolic nucleases restrict the half-life of plasmid DNA to 50-90 min. ${ }^{38}$ Interestingly, even recombinant viruses are inhibited by intracellular degradation. In polarised cells, the AAV capsid is ubiquinated and subsequently enters the ubiquitin proteasome-dependent degradation pathway. ${ }^{39,40}$ Thus, proteasome inhibitors augmented AAV2mediated gene transfer in the mouse lung from undetectable levels to $10 \%$ of airway epithelial cells transfected. ${ }^{39}$

In airway epithelial cells the nuclear membrane is an important barrier for non-viral gene transfer. ${ }^{41}$ It is currently uncertain if strategies, such as the addition of nuclear localisation signals ${ }^{42}$ or lactosylated poly-Llysine, ${ }^{43}$ which increased nuclear transfection in other cell types, will be beneficial in these.

Although, it has been demonstrated that AAV is degraded by proteasomes, non-viral GTAs are probably more affected by intracellular degradation and nuclear entry than viruses. Thus, new strategies are urgently required to overcome the intracellular barriers. It is also important to note, that these barriers are likely to be celltype specific and therefore studies need to be carried out in the 'relevant' cell type.

\section{Expression cassettes have been improved to enable prolonged trangene expression}

Current non-integrating viral and non-viral GTAs are unable to maintain sustained expression. Immuno-suppressants have been reported to prolong transgene expression, ${ }^{44}$ due to their ability to reduce the $\mathrm{T}$ cellmediated response. In addition, Scaria et al showed that incorporation of the adenoviral gene ICP47 into an adenoviral vector, which reduced MHC class I antigen presentation in $\mathrm{CD}^{+} \mathrm{T}$ cells, prolonged expression in primate lung up to 21 days. ${ }^{45}$

The strong CMV promoter has traditionally been used in most GTAs. However, more recently evidence has been provided that weaker eukaryotic or hybrid promoters, such as the polyubiquitin $\mathrm{C}$ promoter, ${ }^{46}$ the elongation factor $1 \alpha$ promoter $^{46}$ and the CMV-Ubiquitin $B$ hybrid promoter ${ }^{47}$ enable prolonged transgene expression. In addition, there is growing evidence that genomic sequences, either within or flanking the gene, might be essential to provide in vivo long-term expression. ${ }^{48}$ Alternative strategies to prolong transgene expression include the development of artificial chromosomes and other self-replicating systems. Huertas et al have developed a circular yeast artificial chromosome (YAC) carrying the human CFTR sequence and the oriP and $E B N A-1$ genes from Epstein-Barr (EBV) virus. ${ }^{49}$ However, it remains to be established if these large constructs are able to transfect airway epithelial cells in vivo.

\section{Intravenous and in utero gene delivery routes have been evaluated}

As noted above topical gene transfer to the lung is severely affected by many extra-cellular barriers. In an attempt to overcome these barriers GTAs or oligonucleotides (ODN) have been administered intravenously (i.v.). It is likely that i.v. administered GTA have to reach the bronchial circulation and avoid lodging in the pulmonary circulation to reach relevant target cells for CF therapy. Fox et al have recently demonstrated that 'naked' ODN are able to leave the bronchial circulation and transfect the cytoplasm, but not the nuclei of airway epithelial cells. ${ }^{50}$ In addition, Kohler et al have shown that i.v. injection of lipid-complexed $\beta$-galactosidase plasmids lead to expression in bronchial epithelium and tracheal submucosal glands of mice. However, this was only achieved with some, but not all of the cationic lipids tested. ${ }^{51}$ Importantly, in the past it has sometimes been difficult to discriminate recombinant from endogenous $\beta$-galactosidase expression and it is therefore important for these potentially exciting findings to be expanded upon.

The possibility for in utero gene therapy for CF has also been investigated. Injection of GTAs into the amniotic fluid, would provide contact with most relevant target sites for CF (pulmonary, gastrointestinal and sinus epithelium). Injection of adenovirus, retrovirus and more recently $\mathrm{AAV}^{52}$ resulted in reporter gene expression in both pulmonary and gastrointestinal epithelium with persistence of transgene expression in the lung ranging from 14 to 30 days after infection. The possibility that in utero gene therapy would tolerise recipients to viral GTAs, has recently been ruled out. ${ }^{53}$ Larson et al have postulated a role for CFTR during development and provided preliminary evidence that transient expression of 
CFTR cDNA in utero alleviates the intestinal defect in the CF knockout mouse long term. ${ }^{54,55}$ These controversial findings, if replicated, may have far reaching implications for traditional gene therapy approaches, as well as other areas of CF research.

\section{Genomic gene repair, mRNA trans-splicing and antisense approaches have been introduced for $\mathrm{CF}$}

Gene repair of the endogenous CFTR gene has two major advantages over traditional gene therapy. If successful, gene repair will ensure gene expression for the lifetime of the cells and appropriate control of gene expression will be guaranteed because the endogenous CFTR promoter is utilised. Preliminary results indicated that the genomic CFTR locus could be modified in primary rat hepatocytes using chimeraplasts (DNA/RNA hybrid oligonucleotides). ${ }^{56}$ Hepatocytes have previously been shown to be easily amenable for gene repair strategies, most likely due to efficient uptake of repair molecules into the nucleus. In addition, a similar approach using small fragment homologous recombination (SFHR) was able to reintroduce the wild-type CFTR sequence into the lungs of CF knockout mice, albeit at very low frequency ${ }^{57}$ However, the specificity of targeted gene repair is currently unknown.

Down-regulation of gene expression through antisense molecules may be of therapeutic benefit in CF. Lambert et al showed that antisense inhibition of the B cell antigen receptor-associated protein (BAP) 31 increased expression of both wild-type CFTR and mutant CFTR and partially restored CFTR chloride channel function. ${ }^{58}$ The exact function of BAP31 is unclear, although the authors speculated that the protein may be involved in retaining mutant CFTR in the ER. Several other chaperone proteins, mucins or the epithelial sodium channel (ENaC), which is up-regulated in CF may be suitable candidates for antisense strategies.

Spliceosome-mediated trans-splicing (SMaRT) has recently been introduced as a means to generate wildtype CFTR mRNA in CF xenograft models. Cells were transfected with very high titres of adenovirus that produced so-called pre-therapeutic wild-type CFTR mRNA molecules (PTMs), which are designed to promote transsplicing with the endogenous CFTR mRNA and $22 \%$ of wild-type CFTR function could be restored. ${ }^{59}$ Similar to gene repair, SMaRT ensures cell-type specific expression of wild-type CFTR mRNA, however efficiency and specificity of the reaction require further improvements, before clinical trials can be considered.

\section{Prospects}

Together with traditional GTAs, new approaches are being developed in order to increase gene transfer efficiency to the airway epithelium. Gersting et $a^{60}$ recently reported that application of a magnetic field to human primary airway epithelial cells transfected with plasmid DNA mixed with superparamagnetic nanoparticles (magnetofection) resulted in more than 100-fold increase in gene transfer. Stem cell-based gene therapy approaches are also being investigated. Two strategies can be envisaged: either targeting lung-resident stem cells with integrating vectors or engineering bone marrow/embryonic cells ex vivo and inducing engraftment into the airway epithelium. Thus, bone marrow-derived stem cells have recently been observed to diferentiate into airway epithelial cells in the lung. ${ }^{61}$ Prolonged and sustained CFTR expression may not be beneficial, especially if integrating vectors are used. To this end, epithelium-specific regulated cassettes are being developed. ${ }^{62}$ Each of the vectors and technologies will have to be tested in relevant in vivo models. Ideally, in contrast to CF mice, these would reproduce the lung pathology seen in CF patients. For this reason efforts are being made to develop a CF ferret ${ }^{63}$ and a CF sheep, ${ }^{64}$ their lung biology being more similar to humans than the mouse. Finally new imaging techniques to monitor the efficiency of gene transfer in living animals/patients will also have to be developed. In addition to positron emission tomography (PET) and magnetic resonance imaging (MRI)-based strategies, laser-induced fluorescence bronchoscopy ${ }^{65}$ has recently been developed as a way to detect gene expression in a non-invasive way in human airways.

\section{Conclusion}

Although topical gene transfer to the airways for $\mathrm{CF}$ gene therapy is more challenging than originally thought, significant progress has been made in pre-clinical research over the last 2 years to overcome some of the hurdles. Most importantly, the identification of more effective viral and non-viral gene transfer agents for airway gene delivery, development of new delivery routes and most recently alternative strategies are keeping the development of $\mathrm{CF}$ gene therapy well on track.

\section{References}

1 Noone PG et al. Safety and biological efficacy of a lipid-CFTR complex for gene transfer in the nasal epithelium of adult patients with cystic fibrosis. Mol Ther 2000; 1: 105-114.

2 Ruiz FE et al. A clinical inflammatory syndrome attributable to aerosolised lipid-DNA administration in cystic fibrosis. Hum Gene Ther 2001; 12: 751-761.

3 Hyde SC et al. Repeat administration of DNA/liposomes to the nasal epithelium of patients with cystic fibrosis. Gene Therapy 2000; 7: 1156-1165.

4 Perricone MA et al. Aerosol and lobar administration of a recombinant adenovirus to individuals with cystic fibrosis. II. Transfection efficiency in airway epithelium. Hum Gene Ther 2001; 12: 1383-1394.

5 Aitken ML et al. A phase I study of aerosolized administration of $\operatorname{tg} \mathrm{AAVCF}$ to cystic fibrosis subjects with mild lung disease. Hum Gene Ther 2001; 12: 1907-1916.

6 Ferrari $\mathrm{S}$ et al. Mucus altering agents as adjuncts for nonviral gene transfer to airway epithelium. Gene Therapy 2001; 8: 1380-1386

7 Sanders NN, Van Rompaey E, De Smedt SC, Demeester J. Structural alterations of gene complexes by cystic fibrosis sputum. Am J Respir Crit Care Med 2001; 164: 486-493.

8 Perricone MA et al. Inhibitory effect of cystic fibrosis sputum on adenovirus-mediated gene transfer in cultured epithelial cells. Hum Gene Ther 2000; 11: 1997-2008.

9 Virella-Lowell I et al. Inhibition of recombinant adeno-associated virus (rAAV) transduction by bronchial secretions from cystic fibrosis patients. Gene Therapy 2000; 7: 1783-1789.

10 Sanders NN et al. Cystic fibrosis sputum: a barrier to the transport of nanospheres. Am J Respir Crit. Care Med 2000; 162: 1905-1911. 
11 Pickles RJ et al. Retargeting the coxsackievirus and adenovirus receptor to the apical surface of polarized epithelial cells reveals the glycocalyx as a barrier to adenovirus-mediated gene transfer. J Virol 2000; 74: 6050-6057.

12 Wang G et al. Increasing epithelial junction permeability enhances gene transfer to airway epithelia in vivo. Am J Respir Cell Mol Biol 2000; 22: 129-138.

$13 \mathrm{Chu} \mathrm{Q}$ et al. EGTA enhancement of adenovirus-mediated gene transfer to mouse tracheal epithelium in vivo. Hum Gene Ther 2001; 12: 455-467.

14 Man $\mathrm{Y}$ et al. Loss of epithelial integrity resulting from Ecadherin dysfunction predisposes airway epithelial cells to adenoviral infection. Am J Respir Cell Mol Biol 2000; 23: 610-617.

15 Coyne CB, Kelly MM, Boucher RC, Johnson LG. Enhanced epithelial gene transfer by modulation of tight junctions with sodium caprate. Am I Respir Cell Mol Biol 2000; 23: 602-609.

16 Croyle MA, Cheng X, Sandhu A, Wilson JM. Development of novel formulations that enhance adenoviral-mediated gene expression in the lung in vitro and in vivo. Mol Ther 2001; 4: 22-28.

17 Weiss DJ et al. Perfluorochemical liquid enhances adeno-associated virus-mediated transgene expression in lung. Mol Ther 2000; 2: 624-630.

18 Das A, Niven R. Use of perfluorocarbon (Fluorinert) to enhance reporter gene expression following intratracheal instillation into the lungs of Balb/c mice: implications for nebulized delivery of plasmids. J Pharm Sci 2001; 90: 1336-1344.

19 Ziady AG et al. Functional evidence of CFTR gene transfer in nasal epithelium of cystic fibrosis mice in vivo following luminal application of DNA complexes targeted to the serpin-enzyme complex receptor. Mol Ther 2002; 5: 413-419.

20 Jost PJ et al. A novel peptide, THALWHT, for the targeting of human airway epithelia. FEBS Lett 2001; 489: 263-269.

21 Zhang L, Collins PL, Boucher RC, Pickles RJ. Respiratory syncytial virus (RSV) infects ciliated cells of airway epithelium via the lumenal membrane. Pediat Pulmonol 2001; (Suppl. 22) 244

22 Yonemitsu $Y$ et al. Efficient gene transfer to airway epithelium using recombinant Sendai virus. Nat Biotechnol 2000; 18: 970-973.

23 Walters RW et al. Binding of adeno-associated virus type 5 to 2,3-linked sialic acid is required for gene transfer. J Biol Chem 2001; 276: 20610-20616.

24 Zabner J et al. Adeno-associated virus type 5 (AAV5) but not AAV2 binds to the apical surfaces of airway epithelia and facilitates gene transfer. J Virol 2000; 74: 3852-3858.

25 Halbert CL et al. Repeat transduction in the mouse lung by using adeno-associated virus vectors with different serotypes. J Virol 2000; 74: 1524-1532.

26 Duan D, Yue Y, Engelhardt JF. Expanding AAV packaging capacity with trans-splicing or overlapping vectors: a quantitative comparison. Mol Ther 2001; 4: 383-391.

27 Ostedgaard LS et al. CFTR with a partially deleted R domain corrects the cystic fibrosis chloride transport defect in human airway epithelia in vitro and in mouse nasal mucosa in vivo. Proc Natl Acad Sci USA 2002; 99: 3093-3098.

28 Pickles RJ, Hirsh AJ, Gerard RJ, Boucher RC. Retargeting Ad5 fibers to bradykinin receptors expressed on the lumenal surface of human airway epithelium. Pediat Pulmonol 2001; 22: (Suppl.22) 247.

29 Drapkin PT et al. Targeting the urokinase plasminogen activator receptor enhances gene transfer to human airway epithelia. Clin Invest 2000; 105: 589-596.

30 Kreda SM, Pickles RJ, Lazarowski ER, Boucher RC. G-proteincoupled receptors as targets for gene transfer vectors using natural small-molecules ligands. Nat Biotechnol 2000; 18: 635640

31 Wang G et al. Human coronavirus 229E infects polarized airway epithelia from the apical surface. J Virol 2000; 74: 9234-9239.

32 Slepushkin VA et al. Infection of human airway epithelia with H1N1, H2N2 and H3N2 influenza A virus strains. Mol Ther 2001; 3: 395-402.

33 Kobinger GP, Weiner DJ, Yu QC, Wilson JM. Filovirus-pseudo- typed lentiviral vector can efficiently and stably transduce airway epithelia in vivo. Nat Biotechnol 2001; 19: 225-230.

34 Chirmule $\mathrm{N}$ et al. Readministration of adenovirus vector in nonhuman primate lungs by blockade of CD40-CD40 ligand interactions. J Virol 2000; 74: 3345-3352.

$35 \mathrm{Kolb} \mathrm{M}$ et al. Budesonide enhances repeated gene transfer and expression in the lung with adenoviral vectors. Am J Respir Crit Care Med 2001; 164: 866-872.

36 Croyle MA, Chirmule N, Zhang Y, Wilson JM. 'Stealth' adenovirus blunt cell-mediated and humoral immune responses against the virus and allow for significant gene expression upon readministration in the lung. J Virol 2001; 75: 4792-4801.

37 Yew NS et al. Reduced inflammatory responses to plasmid DNA vectors by elimination and inhibition of immunostimulatory CpG motifs. Mol Ther 2000; 1: 255-262.

38 Pollard $\mathrm{H}$ et al. Ca2+-sensitive cytosolic nucleases prevent efficient delivery to the nucleus of injected plasmids. J Gene Med 2001; 3: 153-164.

39 Duan D et al. Endosomal processing limits gene transfer to polarized airway epithelia by adeno-associated virus. J Clin Invest 2000; 105: 1573-1587.

$40 \mathrm{Yan} \mathrm{Z}$ et al. Ubiquitination of both adeno-associated virus type 2 and 5 capsid proteins affects the transduction efficiency of recombinant vectors. J Virol 2002; 76: 2043-2053.

41 Griesenbach U et al. Cytoplasmic deposition of NFKB decoy oligonucleotides is insufficient to inhibit bleomycin induced pulmonary inflammation. Gene Therapy 2002 (in press).

42 Cartier R, Reszka R. Utilization of synthetic peptides containing nuclear localization signals for nonviral gene transfer systems. Gene Therapy 2002; 9: 157-167.

43 Klink DT, Chao S, Glick MC, Scanlin TF. Nuclear translocation of lactosylated poly-L-lysine/cDNA complex in cystic fibrosis airway epithelial cells. Mol Ther 2001; 3: 831-841.

44 Cassivi SD et al. Transplant immunosuppression increases and prolongs transgene expression following adenoviral-mediated transfection of rat lungs. J Heart Lung Transplant 2000; 19: 984994.

45 Scaria A et al. Adenoviral vector expressing ICP47 inhibits adenovirus-specific cytotoxic $\mathrm{T}$ lymphocytes in nonhuman primates. Mol Ther 2000; 2: 505-514.

46 Gill DR et al. Increased persistence of lung gene expression using plasmids containing the ubiquitin $\mathrm{C}$ or elongation factor $1 \alpha$ promoter. Gene Therapy 2001; 8: 1539-1546.

47 Yew NS et al. High and sustained transgene expression in vivo from plasmid vector containing a hybrid ubiquitin promoter. Mol Ther 2001; 4: 75-82.

48 Stoll SM et al. Epstein-Barr virus/human vector provides highlevel, long-term expression of alpha1-antitrypsin in mice. Mol Ther 2001; 4: 122-129.

49 Huertas D, Howe S, McGuigan A, Huxley C. Expression of the human CFTR gene from episomal oriP-EBNA1-YACs in mouse cells. Hum Mol Genet 2000; 9: 617-629.

50 Fox $\mathrm{E}$ et al. Towards nucleic acid transfer to the airway epithelium via the systemic route. Mol Ther 2001; 3: S197.

51 Koehler DR et al. Targeting transgene expression for cystic fibrosis gene therapy. Mol Ther 2001; 4: 58-65.

52 Boyle MP et al. In utero AAV-mediated gene transfer to rabbit pulmonary epithelium. Mol Ther 2001; 4: 115-121.

53 Lipshutz GS, Flebbe-Rehwaldt L, Gaensler KML. Re-expression following readministration of an adenoviral vector in adult mice after initial in utero adenoviral administration. Mol Ther 2000; 2: 374-380.

54 Larson JE et al. Gene transfer into the fetal primate: evidence for the secretion of transgene product. Mol Ther 2000; 2: 631-639.

55 Larson JE et al. CFTR modulates lung secretory cell proliferation and differentiation. Am J Physiol Lung Cell Mol Physiol 2000; 279: L333-L341.

56 Griesenbach U et al. Conversion of wild-type CFTR to the G551D mutation in primary rat hepatocytes using RNA/DNA oligonucleotides. Pediat Pulmonol 2001; (Suppl. 22) 252.

57 Goncz KK et al. Expression of (F508 CFTR in normal lung after 
site-specific modification of CFTR sequences by SFHR. Gene Therapy 2001; 8: 961-965.

58 Lambert $\mathrm{G}$ et al. Control of cystic fibrosis transmembrane conductance regulator expression by BAP31. J Biol Chem 2001; 276: 20340-20345.

59 Liu X et al. Partial correction of endogenous (F508 CFTR in human cystic fibrosis airway epithelia by spliceosome-mediated RNA trans-splicing. Nat Biotechnol 2002; 20: 47-52.

60 Gersting $S$ et al. Magnetofection of permanent and primary human airway epithelial cells. Mol Ther 2001; 3: Abstr. 1017.

61 Krause DS et al. Multi-organ, multi-lineage engraftment by a single bone marrow-derived stem cell. Cell 2001; 105: 369-377.
62 Ye L et al. Regulated expression of the human CFTR gene in epithelial cells. Mol Ther 2001; 3: 723-733.

63 Jiang Q, Li Z, Zhang Y, Engelhardt JF. Development of a ferret model of the cystic fibrosis. Pediat Pulmonol 2000; 20 (Suppl.): Abstr. 164.

64 Mouchel $\mathrm{N}$ et al. The sheep genome contributes to localization of control elements in a human gene with complex regulatory mechanisms. Genomics 2001; 76: 9-13.

65 Rooney C et al. Laser induced fluorescence bronchoscopy for the detection of gene transfer. Mol Ther 2001; 3: Abstr. 1152. 